\title{
Regulation of the downside angular velocity of a drilling string with a P-I controller
}

\author{
A. Terrand-Jeanne, V. Dos Santos Martins, V. Andrieu
}

\begin{abstract}
In this paper, we demonstrate that a simple Proportional Integral controller allow to regulate the angular velocity of a drill-string despite unknown friction torque and by only using measurement of angular velocity at the surface. Our model is an one dimensional damped wave equation subject to an unknown dynamics at one side while one control and measure at the other side. After turning this system of balance law into the Riemann coordinates, we design a Lyapunov functional for proving the exponential stability of the closedloop and show how it implies the regulation of the angular velocity.
\end{abstract}

\section{INTRODUCTION}

In order to find and exploit oil, one needs to dig deeper and deeper into the earth's surface. The first consequence on raising the length of the pipe is the increase of several phenomena causing damage until the break of the device. These unwanted phenomena are mainly characterized by oscillations inside the pipe. These oscillations may appear in axial, radial and lateral directions. According to several studies (i.e [7], [4]), the radial oscillation, namely Stick-Slip phenomena, is the most disturbing one. Indeed, it results on angular deformations traveling along the pipe, leading to important damage and furthermore and it is the source of other oscillating phenomena (Bit-Bounced and lateral oscillation).

From a mathematical point of view, first studies on this topic were based on lumped parameter model as in [4]. Nevertheless, the increase of the length of the pipe forces one to consider a distributed parameter model in order to deal with all the possible oscillations frequencies.

The control theory for such mathematical model is still an active domain of research. Recently, several works exploiting a PDE-based model to stabilize oscillating phenomena have been made. For example, by using a backstepping approach as in ([3]) or a flatness one in ([10]). Another method transforms the whole system in an equivalent time-delay system before ensuring its stability ([7]). Note however, that this transformation is impossible when taking into account a distributed damping along the drill pipe.

The main contribution of this article is the analysis of the closed loop stability when the control is provided in the form of a proportional integral (P-I) feedback depending on the topside angular velocity measurement. This shows that this control regulates the angular velocity of the drill bit to a given reference.

Since the seminal paper of S.A. Pohjolainen in 1982 [8], the problem of output regulation for PDE systems have received a huge interest from the control community. Following this paper, an important effort has been made to consider more general class of PDE and also to relax some crucial assumptions. For instance, it has been shown in [14] that it is possible to relax the compactness requirement on the operator. Moreover, it has been shown in [5] or [15] that it was possible to design a P-I for boundary control for different classes of hyperbollic systems. Following the route of a recent contributions in [13], [11], [2], we prove the regulation and the stabilization employing a Lyapunov approach.

In [3] and [9] the regulation problem of the angular velocity for drilling is also considered. In these works, an observer is built to perform a full state feedback and design a backstepping transformation. To compare, our control design is fairly simple since it is a P-I control law which only needs the surface angular velocity measurement. Moreover, our design methodology employs a novel Lyapunov design which should allow to consider nonlinear terms in the future.

\section{Problem Statement}

\section{A. Regulation of the angular velocity}

We use the following PDE-ODE model describing the mechanical oscillations evolving along the drill string as given in [7] :

$$
\begin{aligned}
\rho J \theta_{t t}(x, t) & =G J \theta_{x x}(x, t)-\beta \theta_{t}(x, t), \\
x \in] 0, L[, t>0 & \\
G J \theta_{x}(0, t) & =c_{a}\left(\theta_{t}(0, t)-\Omega(t)\right) \\
I_{b} \theta_{t t}(L, t) & =-G J \theta_{x}(L, t)-T_{f r}\left(\theta_{t}(L, t)\right)
\end{aligned}
$$

where for all $x$ in $(0, L) \theta(x, t)$ is the angular position of the drill string at point $x$ and time $t$ with respect to a given reference frame. Also, subscripts $t, x, t t, .$. denote the first or second derivative w.r.t variables $t$ or $x . G, J, \rho, \beta, c_{a}$ and $I_{b}$ are positive mechanical parameters and $\Omega(t)$ is the velocity of the rotatory table that we will use as controller. The different parameters are given in Appendix A. $T_{f r}$ : $\mathbb{R} \mapsto \mathbb{R}$ is a function which describes the frictions between the drill bit and the earth. Due to Stribeck effect which occurs at low velocity when the lubrication is not totally effective (see for instance [7]), this function is highly nonlinear for small values of $\theta_{t}(L, t)$. However, when the drill pipe is rotating fast enough, this frictions function becomes linear. Hence, in the sequel we consider that :

$$
T_{f r}\left(\theta_{t}(L, t)\right)=c_{b} \theta_{t}(L, t)+T_{0}
$$

where $c_{b}$ is a real number and $T_{0}$ is also a real number which is assumed to be constant but unknown. 
The measured output of this system is the angular position of the pipe at the top. In other words, we measure the following quantity :

$$
y(t)=\theta_{t}(0, t)
$$

Our control objective is to regulate the velocity at the bottom which is denoted

$$
\underline{y}(t)=\theta_{t}(L, t)
$$

to a prescribed constant reference velocity.

The control we intend to solve is the following. We wish to find a control input $\Omega(t)$ depending only on the measured output such that for every (unknown) constant value of $T_{0}$, the to be regulated output $y$ is regulated to a given constant reference value denoted $\underline{y}_{r}$. The structure of the control law we use to solve this problem is simply a proportionalintegral (P-I) control law. More precisely, the control input is provided by dynamic error feedback modeled by equation of the form :

$$
\Omega(t)=-k_{p}\left[y(t)-y_{r e f}\right]-k_{i} \eta, \dot{\eta}=y(t)-y_{\text {ref }} \forall t \geq 0,
$$

where $\underline{y}_{r}$ is a positive real number which is the constant speed at which we wish the velocity at the bottom of the pipe to be regulated. In the following we give sufficient conditions on the parameters $G, J, \rho, \beta, c_{a}$ and $I_{b}$ such that there exist $k_{p}$ and $k_{i}$ which ensure the exponential stability of the equilibrium and regulation. In other words, along the solutions of the system (1) with the control input (5) the equilibrium has to be exponentially stable and also we need to have

$$
\lim _{t \rightarrow+\infty}\left|\underline{y}(t)-y_{\text {ref }}\right|=0
$$

In a first part of the paper, we rewrite the model in Riemannian coordinates. Defining the state space solution and its topology we give our main result which shows that regulation is obtained by our P-I control law. The remaining part of the paper is devoted to show this result. The proof, starts by showing that the regulation property is implied by the exponential stability of the equilibrium state of the closed loop systems. To show that the equilibrium state is exponentially stable, we construct a Lyapunov functional.

\section{B. Riemannian coordinates:}

The first step of our study is to rewrite the drilling model given in mechanical coordinates in system (1)-(2)-(3)-(4) in closed loop with the control law (5) into a normalized Riemann coordinates. In [2], authors give a general method to reduce linear hyperbolic systems of conservation law with ODE at its boundaries in first order transport equation coupled with ODE via theirs boundaries condition. Note however, that in eq. (1), $\beta \neq 0$, hence, we are dealing with systems of balance law. If the method remains similar, the resulting transport equations are coupled with each other. For $x$ in $(0,1)$ and $t \geq 0$ let :

$$
\begin{aligned}
\varphi^{-}(x, t) & =\left[\theta\left(L x, \frac{L t}{c}\right)\right]_{t}-\left[\theta\left(L x, \frac{L t}{c}\right)\right]_{x}, \\
\varphi^{+}(x, t) & =\left[\theta\left(L x, \frac{L t}{c}\right]_{t}+\left[\theta\left(L x, \frac{L t}{c}\right)\right]_{x},\right. \\
z(t) & =\left[\theta\left(L, \frac{L t}{c}\right)\right]_{t}, \\
\xi(t) & =\frac{2 L}{c} \eta(t),
\end{aligned}
$$

with $c^{2}=\frac{G}{\rho}$. Employing equations (1)-(2)-(3)-(4) and (5), this implies for all $t \geq 0$ :

$$
\begin{aligned}
\varphi_{t}(x, t) & =\left[\begin{array}{cc}
-\partial_{x}-\frac{\lambda}{2} & -\frac{\lambda}{2} \\
-\frac{\lambda}{2} & \partial_{x}-\frac{\lambda}{2}
\end{array}\right] \varphi(x, t), \forall x \in(0,1), \\
z_{t}(t) & =-(a+b) z(t)+a \varphi^{-}(1, t)+d \\
\xi_{t}(t) & =\varphi^{-}(0, t)+\varphi^{+}(0, t)-\tilde{y}_{r e f}
\end{aligned}
$$

with the boundary conditions :

$$
\begin{aligned}
& \varphi^{-}(0, t)=\alpha_{0} \varphi^{+}(0, t) \\
& \quad+K_{p}\left(\varphi^{-}(0, t)+\varphi^{+}(0, t)-\tilde{y}_{r e f}\right)+K_{i} \xi(t), \\
& \varphi^{+}(1, t)=-\varphi^{-}(1, t)+2 z(t),
\end{aligned}
$$

with $\varphi(x, t)=\left(\begin{array}{l}\varphi^{-}(x, t) \\ \varphi^{+}(x, t)\end{array}\right)$ and the normalized parameters are given as :

$$
\begin{aligned}
& \lambda=\frac{\beta L}{c \rho J}, \alpha_{0}=\frac{G J-c_{a} c}{G J+c_{a} c} \\
& a=\frac{G J L}{I_{b} c^{2}}, b=\frac{c_{b} L}{c I_{b}}, d=-\frac{T_{0} L^{2}}{I_{b} c^{2}},
\end{aligned}
$$

the normalized reference and the normalized output to regulate are respectively :

$$
\tilde{y}_{r e f}=\frac{c y_{r e f}}{2 L}, \underline{\tilde{y}}(t)=\varphi^{-}(1, t)+\varphi^{+}(1, t)=2 z(t)
$$

and the normalized P-I gains are given as :

$$
K_{p}=\frac{c_{a} c}{G J+c_{a} c} k_{p}, \quad K_{i}=\frac{c_{a} c}{G J+c_{a} c} k_{i} .
$$

Equations (11), (12), (13) with boundary conditions (14) (15) define a hyperbolic partial differential equation coupled at the boundaries with two external ordinary differential equations. The state space denoted by $\mathbb{X}$ is the Hilbert space defined as :

$$
\mathbb{X}=\left(L^{2}(0,1)\right)^{2} \times \mathbb{R}^{2},
$$

equipped with the norm defined for $v=\left(\varphi^{-}, \varphi^{+}, z, \xi\right)$ in $\mathbb{X}$ as :

$$
\|v\|_{\mathbb{X}}=\left\|\varphi^{-}\right\|_{L^{2}(0,1)}+\left\|\varphi^{+}\right\|_{L^{2}(0,1)}+|z|+|\xi| .
$$

We introduce also a smoother state space defined as :

$$
\mathbb{X}_{1}=\left(H^{1}(0,1)\right)^{2} \times \mathbb{R}^{2},
$$

As it has been shown in [2], for each initial condition $v_{0}$ in $\mathbb{X}$ which satisfies the boundary conditions (14) and (15), there exists a unique weak solution that we denoted $v$ and which belongs to $C^{0}([0,+\infty) ; \mathbb{X})$. Moreover, if the initial condition 
$v_{0}$ satisfies also the $C^{1}$-compatibility condition (see [2] for more details) and lies in $\mathbb{X}_{1}$ then the solution is strong, and belongs to :

$$
v \in C^{0}\left([0,+\infty) ; \mathbb{X}_{1}\right) \cap C^{1}([0,+\infty) ; \mathbb{X})
$$

\section{Main result}

With all these preliminaries, we are now able to state our main result.

Theorem 1 (Regulation and stabilization): For all $\left(\alpha_{0}, \lambda\right.$, $a, b)$ in $\mathbb{R}^{4}$ with $0 \leq \lambda<2, a>0, b \geq 0$ there exist real numbers $K_{i}, K_{p}$ and positive real numbers $k$ and $\nu$ such that for all constant references $\tilde{y}_{r e f}$, all unknown $d$ and all initial conditions in $\mathbb{X}$ the following holds.

1) There exists an equilibrium state denoted $v_{\infty}$ which is globally exponentially stable in $\mathbb{X}$ for system (11)-(15). More precisely, we have :

$$
\left\|v(t)-v_{\infty}\right\|_{\mathbb{X}} \leq k \exp (-\nu t)\left\|v_{0}-v_{\infty}\right\|_{\mathbb{X}}
$$

2) If moreover $v_{0}$ satisfies the $C^{1}$-compatibility condition and is in $\mathbb{X}_{1}$, the regulation is achieved, i.e.

$$
\lim _{t \rightarrow+\infty}\left|\underline{\tilde{y}}(t)-\tilde{y}_{r e f}\right|=0 .
$$

As in [3] or [9], we solve the regulation problem around the equilibrium state by acting at the opposed boundary. Advantages of our approach is that we control the rotatory table and not directly the quantity $\theta_{x}(0, t)$ and that we only use $\theta_{t}(0, t)$ for designing our controller.

The stability analysis of this kind of models has been considered in [11], [2]. The dynamics at the top side boundary is due to the integral action of the control law. The stability analysis of PDE coupled with integral action has been initiated by [8] for parabolic systems (see also [15] for hyperbolic systems) following a spectral analysis. Recently, an analysis for $2 \times 2$ hyperbolic PDE has been performed with a Lyapunov approach in [12]. Our approach follows this route. Note however that it is not a direct application of this result due to the other dynamics boundary and that (11) is a system of balance laws and not a system of conservation laws (see [2]).

From a practical point of view, it may be not interesting to ask too much to the proportional part of the control. For instance, and as it has been shown in [1], canceling the reflexion when $\left|\alpha_{0}\right| \leq 1$ with a proportional gain $K_{p}$, may not be an interesting approach due to lack of robustness with respect to input delays. In the following corollary ${ }^{1}$, we show that if $\left|\alpha_{0}\right|<1$ then a proportional part is not necessary to obtain regulation.

Corollary 1 (Regulation for stable systems): Assume $\left|\alpha_{0}\right|<1$, then for all $(\lambda, a, b)$ in $\mathbb{R}^{3}$ with $0 \leq \lambda<2$, $a>0, b \geq 0$, then picking $K_{p}=0$, there exist $K_{i}$ in $\mathbb{R}$ and positive real numbers, $k$ and $\nu$ such that the conclusion of the Theorem 1 holds.

\footnotetext{
${ }^{1}$ The proof of this corollary is deduced from the following Lyapunov analysis picking $\alpha_{p}=\alpha_{0}$.
}

\section{Proof OF THEOREM 1}

\section{A. Stabilization implies regulation}

In this first subsection, we explicitly give the equilibrium state of the system (11), (12), (13) with the boundary conditions (14) and (15). We show also that if we assume that $K_{p}$ and $K_{i}$ are selected such that this equilibrium point is exponentially stable along the closed loop, then the regulation is achieved.

If we denote $v_{\infty}=\left(\varphi_{\infty}, z_{\infty}, \xi_{\infty}\right)$ the equilibrium states of (11)-(15), we obtain :

$$
\begin{aligned}
& z_{\infty}=\frac{\tilde{y}_{r e f}}{2} \\
& \xi_{\infty}=\frac{\left(1+\alpha_{0}\right)(a(1+\lambda)+b)-2 a \alpha_{0}}{2 a K_{i}} \tilde{y}_{r e f}-\frac{1+\alpha_{0}}{a K_{i}} d, \\
& \varphi_{\infty}^{-}(x)=-\frac{\lambda}{2} \tilde{y}_{r e f} x+\frac{1}{1+\alpha_{0}}\left(\alpha_{0} \tilde{y}_{r e f}+K_{i} \xi_{\infty}\right), \\
& \varphi_{\infty}^{+}(x)=\frac{\lambda}{2} \tilde{y}_{r e f} x+\frac{\tilde{y}_{r e f}}{1+\alpha_{0}}-\frac{K_{i}}{1+\alpha_{0}} \xi_{\infty} .
\end{aligned}
$$

In the following, we first show that this regulation problem can be rephrased as a stabilization of an equilibrium state.

Proposition 1: Assume that there exists a functional $W$ : $\mathbb{X} \rightarrow \mathbb{R}_{+}$, and positive real numbers $\omega$ and $L$ such that :

$$
\frac{\left\|v_{\infty}-v\right\|_{\mathbb{X}}^{2}}{L} \leq W(v) \leq L\left\|v_{\infty}-v\right\|_{\mathbb{X}}^{2} .
$$

Assume moreover that for all $v_{0}$ in $\mathbb{X}$ and all $t_{0}$ in $\mathbb{R}_{+}$such that the solution $v$ of (11)-(15) initiated from $v_{0}$ is $C^{1}$ at $t=t_{0}$, we have :

$$
\dot{W}(v(t)) \leq-\omega W(v(t)) .
$$

Then points 1) and 2) of Theorem 1 hold.

Proof: The proof of point 1) is by now standard. Let $v_{0}$ be in $\mathbb{X}_{1}$ and satisfies the $C^{0}$ and $C^{1}$-compatibility conditions. It yields that $v$ is smooth for all $t$. Consequently, (23) is satisfied for all $t \geq 0$. With Grönwall lemma, this implies that :

$$
W(v(t)) \leq e^{-\omega t} W\left(v_{0}\right) .
$$

Hence with (22), this implies that (20) holds with $k=L$ and $\nu=\frac{\omega}{2}$ for initial conditions in $\mathbb{X}_{1} \cdot \mathbb{X}_{1}$ being dense in $\mathbb{X}$, the result holds also with initial condition in $\mathbb{X}$ and point 1) is satisfied.

Let show point 2). With the definition of $\underline{y}(t)$ in (18) and the definition of the equilibrium, we have :

$$
\underline{\tilde{y}}(t)-\tilde{y}_{r e f}=\varphi^{-}(1, t)+\varphi^{+}(1, t)-\varphi_{\infty}^{-}(1)-\varphi_{\infty}^{+}(1) .
$$

Moreover, if we consider the dynamics of $\ell=\left(\varphi_{x}, z, \xi\right)$, it can be shown that if we pick initial condition $v_{0}$ in $\mathbb{X}_{1}$ which satisfies $C^{1}$ compatibility condition, $\ell$ is in $\mathbb{X}$ and satisfies the dynamics (11)-(15). Hence, $\|\ell\|_{\mathbb{X}}$ converge to 0 . It implies that along solutions $\|v\|_{\mathbb{X}_{1}}$ converges also to 0 . Since with Sobolev embedding

$$
\begin{aligned}
\sup _{x \in[0,1]}\left|\varphi(x, t)-\varphi_{\infty}(x)\right| & \leq C\|\varphi(\cdot, t)\|_{H^{1}(0,1)}, \\
& \leq C\|v(t)\|_{\mathbb{X}_{1}},
\end{aligned}
$$


where $C$ is a positive real number, it implies :

$$
\lim _{t \rightarrow+\infty}\left|\varphi^{-}(1, t)+\varphi^{+}(1, t)-\varphi_{\infty}^{-}(1)-\varphi_{\infty}^{+}(1)\right|=0 .
$$

Consequently, (21) holds and point 2) is satisfied.

With this proposition in hand and since the system is linear, it turns out that to prove the Theorem 1, it is sufficient to construct a Lyapunov functional. This property doesn't depend on the value of $y_{\text {ref }}$ and the unknown parameter $d$. So in the following, it is assumed that $y_{r e f}=d=0$ and a Lyapunov functional is designed.

\section{B. Lyapunov functional construction}

1) Step 1, Let forget the $\xi$ dynamics: In this subsection we construct a Lyapunov function for the system (11)-(15) without taking into account the integral part which will be added in the next subsection. So here, we consider this PDE system :

$$
\begin{aligned}
\varphi_{t}(x, t) & =\left[\begin{array}{cc}
-\partial_{x}-\frac{\lambda}{2} & -\frac{\lambda}{2} \\
-\frac{\lambda}{2} & \partial_{x}-\frac{\lambda}{2}
\end{array}\right] \varphi(x, t), \forall x \in(0,1), \\
z_{t}(t) & =-(a+b) z(t)+a \varphi^{-}(1, t),
\end{aligned}
$$

with the boundary conditions :

$$
\begin{aligned}
& \varphi^{-}(0, t)=\alpha_{0} \varphi^{+}(0, t)+K_{p}\left(\varphi^{-}(0, t)+\varphi^{+}(0, t)\right)+K_{i} \xi(t), \\
& \varphi^{+}(1, t)=-\varphi^{-}(1, t)+2 z(t),
\end{aligned}
$$

Let $V: L^{2}(0,1)^{2} \times \mathbb{R} \mapsto \mathbb{R}_{+}$be the function defined by :

$$
\begin{aligned}
V(\varphi, z)=q z^{2}+\int_{0}^{1} \varphi^{-}(x)^{2} & e^{-\mu x} d x \\
& +\int_{0}^{1} p \varphi^{+}(x)^{2} e^{\mu x} d x
\end{aligned}
$$

which is well defined. With a slight abuse of notation, we write $V(t)=V(\varphi(\cdot, t), z(t))$ and we denote by $\dot{V}(t)$ the time derivative of the Lyapunov function along solutions which are $C^{1}$ in time.

Proposition 2: For all $\left(\lambda, a, b, \alpha_{0}\right)$ with $2>\lambda>0, a>0$ and $b \geq 0$ there exist positive real numbers $K_{p}, p, \mu, q, \omega_{1}$ and $\omega_{2}$ such that along $C^{1}$ solution of the system (25) and (26) :

$$
\dot{V}(t) \leq-\omega_{1} V(t)+\nu|\xi(t)|^{2}
$$

Proof: Let

$$
V(t)=V_{1}(t)+V_{2}(t)
$$

with

$$
\begin{aligned}
& V_{1}(t)=q z^{2}(t), \\
& V_{2}(t)=\int_{0}^{1}\left(\varphi^{-}(x, t)\right)^{2} e^{-\mu x} d x+\int_{0}^{1} p\left(\varphi^{+}(x, t)\right)^{2} e^{\mu x} d x .
\end{aligned}
$$

The time derivative of (29) along the system (25) with the boundary condition (26) can be written for all $\mu_{1}$ and $\mu_{2}$ in
$\mathbb{R}:$

$$
\begin{array}{r}
\dot{V}(t)=-\mu_{2} V_{1}(t)-\left(\mu-\mu_{1}\right) V_{2}(t)-w_{0}(t)^{T} \mathcal{P} w_{0}(t) \\
-\int_{0}^{1} \varphi(x, t)^{T} \mathcal{N}\left(\mu_{1}\right) \varphi(x, t) d x \\
-w_{1}(t)^{T} \mathcal{M}\left(\mu, \mu_{2}\right) w_{1}(t),
\end{array}
$$

where :

$w_{0}(t)=\left(\varphi^{+}(0, t) \quad K_{i} \xi(t)\right)^{T}, w_{1}(t)=\left(\varphi^{-}(1, t) \quad z(t)\right)^{T}$,

and :

$$
\begin{aligned}
\mathcal{M} & =\left[\begin{array}{cc}
e^{-\mu}-p e^{\mu} & 2 p e^{\mu}-a q \\
2 p e^{\mu}-a q & \left(2(a+b)-\mu_{2}\right) q-4 p e^{\mu}
\end{array}\right], \\
\mathcal{N} & =\left[\begin{array}{cc}
\left(\mu_{1}+\lambda\right) e^{-\mu x} & \lambda \frac{e^{-\mu x}+p e^{\mu x}}{2} \\
\lambda \frac{e^{-\mu x}+p e^{\mu x}}{2} & p\left(\mu_{1}+\lambda\right) e^{\mu x}
\end{array}\right], \\
\mathcal{P} & =\left[\begin{array}{cc}
p-\alpha_{p}^{2} & \alpha_{p} \\
\alpha_{p} & -1
\end{array}\right],
\end{aligned}
$$

where :

$$
\alpha_{p}=\frac{\alpha_{0}+K_{p}}{1-K_{p}} .
$$

First of all remark that if :

$$
p-\alpha_{p}^{2}>0
$$

then there exists a positive real number $\nu$ such that :

$$
-w_{0}(t)^{T} \mathcal{P} w_{0}(t) \leq \nu\left|K_{i} \xi(t)\right|^{2} .
$$

On top of that, if :

$$
\mu_{2}>0,\left(\mu-\mu_{1}\right)>0, \mathcal{M} \geq 0, \mathcal{N} \geq 0
$$

then the candidate Lyapunov functional satisfies : (28).

Let us first show that we can find the parameters such that (32) are satisfied. Consider the mapping $\left(s_{1}, s_{2}, \mu\right) \mapsto$ $F\left(s_{1}, s_{2},\right)$ given by :

$$
F\left(s_{1}, s_{2}, \mu\right)=\sqrt{s_{1}}\left(\lambda+s_{2}\right)-\frac{\lambda}{2}\left(1+s_{1} e^{\mu}\right) .
$$

Since $\lambda<2$, picking $\mu$ sufficiently small, $0<\mu<1$, the following inequality is always true :

$$
F\left(e^{-2 \mu}, \mu, \mu\right)=e^{-\mu}(\lambda+\mu)-\frac{\lambda}{2}\left(1+e^{-\mu}\right)>0 .
$$

So, let $p$ and $\mu_{1}$ be positive real numbers such that :

$$
0<p<e^{-2 \mu}, \mu_{1}<\mu,
$$

and $F\left(p, \mu_{1}, \mu\right)>0$ which exists by continuity of the mapping $F$. Note that :

$$
\begin{aligned}
\operatorname{det}(\mathcal{N}) & =p\left(\lambda+\mu_{1}\right)^{2}-\frac{\lambda^{2}}{4}\left(e^{-\mu x}+p e^{\mu x}\right)^{2} \\
& >F\left(p, \mu_{1}, \mu\right)\left[\sqrt{p}\left(\lambda+\mu_{1}\right)+\frac{\lambda}{2}\left(e^{-\mu x}+p e^{\mu x}\right)\right], \\
& >0 .
\end{aligned}
$$

So, $\mathcal{N} \geq 0$. 
On another side, $\mathcal{M} \geq 0$ if and only if :

$$
\begin{aligned}
& e^{-\mu}-p e^{\mu} \geq 0 \\
& f(q) \geq 0
\end{aligned}
$$

where :

$$
\begin{aligned}
f(q) & =\left(e^{-\mu}-p e^{\mu}\right)\left(q\left(2(a+b)-\mu_{2}\right)-4 p e^{\mu}\right) \\
& -\left(2 p e^{\mu}-a q\right)^{2} \\
= & -a^{\prime} q^{2}+b^{\prime} q-c^{\prime},
\end{aligned}
$$

and $a^{\prime}, b^{\prime}$ and $c^{\prime}$ are positives real numbers given as :

$$
\begin{aligned}
a^{\prime} & =a^{2} \\
b^{\prime} & =\left(e^{-\mu}-p e^{\mu}\right)\left(2(a+b)-\mu_{2}\right)+4 p a e^{\mu} \\
c^{\prime} & =\left(e^{-\mu}-p e^{\mu}\right) 4 p e^{\mu}+4 p^{2} e^{2 \mu}=4 p
\end{aligned}
$$

This function $f(q)$ is a second order polynomial, which maximum is reached for $q=\frac{b^{\prime}}{2 a^{\prime}}$. In this case, $f(q)$ is strictly positive if and only if :

$$
b^{\prime 2}-4 a^{\prime} c^{\prime}=\left(b^{\prime}-2 \sqrt{a^{\prime} c^{\prime}}\right)\left(b^{\prime}+2 \sqrt{a^{\prime} c^{\prime}}\right)>0 .
$$

Since $a^{\prime}, b^{\prime}$ and $c^{\prime}$ are positives, it remains to verify that $b^{\prime}-2 \sqrt{a^{\prime} c^{\prime}}$ is positive. Note that keeping in mind that $\left(e^{-\mu}-\right.$ $\left.p e^{\mu}\right)>0$, it yields :

$$
\begin{aligned}
\frac{b^{\prime}-2 \sqrt{a^{\prime} c^{\prime}}}{\left(e^{-\mu}-p e^{\mu}\right)} & =\left(2(a+b)-\mu_{2}\right)-\frac{4 a\left(\sqrt{p}-p e^{\mu}\right)}{e^{-\mu}-p e^{\mu}} \\
& =2 a\left(1-\frac{2 \sqrt{p}}{e^{-\mu}+\sqrt{p}}\right)+2 b-\mu_{2}
\end{aligned}
$$

Since $\frac{2 \sqrt{p}}{e^{-\mu}+\sqrt{p}}<1$, we can select $\mu_{2}>0$ sufficiently small such that $f(q)>0$, so $\mathcal{M} \geq 0$.

Finally, we pick $K_{p}$ such that (31) holds which achieves the proof.

2) Step 2, adding the integral part: In this section, the integral parameter $K_{i}$ is tuned based on the construction of a Lyapunov functional. Let $: W: \mathbb{X} \mapsto \mathbb{R}_{+}$be the function defined by :

$$
W(\varphi, z, \xi)=V(\varphi, z)+r U(\xi, \varphi, z)^{2},
$$

where :

$$
U(\xi, \varphi, z)=\xi+m^{\top} M(\varphi)+n z,
$$

where $r>0, m=\left(m_{1}, m_{2}\right)$ is a vector in $\mathbb{R}^{2}$ and $n$ a real number that will be selected later and $M: L^{1}(0,1) \mapsto \mathbb{R}^{2}$ is an operator defined as :

$$
M(\varphi)=\int_{0}^{1}(I+R x) \varphi(x) d x
$$

with :

$$
R=\left[\begin{array}{ll}
\frac{\lambda}{2} & -\frac{\lambda}{2} \\
\frac{\lambda}{2} & -\frac{\lambda}{2}
\end{array}\right]
$$

Again, with a slight abuse of notation, we write $W(t)=$ $W(\varphi(\cdot, t), z(t), \xi(t))$ and we denote by $\dot{W}(t)$ the time derivative of the Lyapunov function along solutions which are $C^{1}$ in time.
Proposition 3: Assume $K_{p}, \lambda, a, b, \alpha_{0}, p, \mu, q$ and $\omega_{1}$ are given such that equation (28) is satisfied, then there exist $r, m_{1}, m_{2}, n$ and $K_{i}$ such that :

$$
\dot{W}(t) \leq-\omega_{2} W(t) .
$$

Proof: First of all, note that :

$$
R\left[\begin{array}{ll}
-\frac{\lambda}{2} & -\frac{\lambda}{2} \\
-\frac{\lambda}{2} & -\frac{\lambda}{2}
\end{array}\right]=0 .
$$

Hence, we get the property :

$$
\begin{aligned}
\dot{M}(t)= & \int_{0}^{1}(I+R x) \varphi_{t}(x, t) d x \\
= & \int_{0}^{1}(I+R x)\left[\begin{array}{cr}
-1 & 0 \\
0 & 1
\end{array}\right] \varphi_{x}(x, t) \\
& +\left[\begin{array}{cc}
-\frac{\lambda}{2} & -\frac{\lambda}{2} \\
-\frac{\lambda}{2} & -\frac{\lambda}{2}
\end{array}\right] \varphi(x, t) d x
\end{aligned}
$$

With an integration by parts, it yields :

$$
\dot{M}(t)=\left[\begin{array}{cc}
-1-\frac{\lambda}{2} & -\frac{\lambda}{2} \\
-\frac{\lambda}{2} & 1-\frac{\lambda}{2}
\end{array}\right] \varphi(1, t)-\left[\begin{array}{cc}
-1 & 0 \\
0 & 1
\end{array}\right] \varphi(0, t),
$$

and consequently,

$$
\begin{aligned}
\dot{U}(t) & =\varphi^{-}(0, t)+\varphi^{+}(0, t)+ \\
m_{1} & \left(\varphi^{-}(0, t)-\left(1+\frac{\lambda}{2}\right) \varphi^{-}(1, t)-\frac{\lambda}{2} \varphi^{+}(1, t)\right) \\
+m_{2}\left(-\frac{\lambda}{2} \varphi^{-}(1, t)+\right. & \left.\left(1-\frac{\lambda}{2}\right) \varphi^{+}(1, t)-\varphi^{+}(0, t)\right) \\
& -n(a+b) z(t)+n a \varphi^{-}(1, t)
\end{aligned}
$$

Employing the boundary conditions (26) and $\alpha_{p}$ defined in (30), it yields :

$$
\begin{gathered}
\dot{U}(t)=\varphi^{-}(1, t)\left(a n-m_{1}\left(1+\frac{\lambda}{2}\right)-m_{2} \frac{\lambda}{2}-\frac{n(a+b)}{2}\right) \\
+\varphi^{+}(1, t)\left(m_{2}\left(1-\frac{\lambda}{2}\right)-m_{1} \frac{\lambda}{2}-\frac{n(a+b)}{2}\right) \\
+\varphi^{+}(0, t)\left(\alpha_{p}+1+m_{1} \alpha_{p}-m_{2}\right) \\
+\xi(t) \frac{K_{i}}{1-K_{p}}\left(1+m_{1}\right) .
\end{gathered}
$$

Our aim is now to solve in $m_{1}, m_{2}$ and $n$ the system :

$$
\left[\begin{array}{ccc}
-1-\frac{\lambda}{2} & -\frac{\lambda}{2} & \frac{a-b}{2} \\
-\frac{\lambda}{2} & 1-\frac{\lambda}{2} & -\frac{a+b}{2} \\
\alpha_{p} & -1 & 0
\end{array}\right]\left[\begin{array}{c}
m_{1} \\
m_{2} \\
n
\end{array}\right]=\left[\begin{array}{c}
0 \\
0 \\
-\alpha_{p}-1
\end{array}\right]
$$

It is possible with :

$$
\begin{aligned}
n & =\frac{2\left(\alpha_{p}+1\right)}{\alpha_{p}(a(\lambda-1)+b)+a \lambda+a+b}, \\
m_{2} & =\frac{+2 a \alpha_{p}}{\alpha_{p}(a(\lambda-1)+b)+a \lambda+a+b}+1, \\
m_{1} & =\frac{2 a}{\alpha_{p}(a(\lambda-1)+b)+a \lambda+a+b}-1 .
\end{aligned}
$$


It can be noticed that since $0 \leq \lambda<2$ and $a+b>0$, these parameters are well defined. In that case, it yields :

$$
\dot{U}(t)=\xi(t) \frac{K_{i}}{1-K_{p}}\left(1+m_{1}\right) .
$$

So we select $K_{i}$ such that :

$$
\frac{K_{i}}{1-K_{p}}\left(1+m_{1}\right)<0 .
$$

Hence, we get :

$$
\begin{aligned}
\frac{2 U(t) \dot{U}(t)}{\left|K_{i}\right|} & \leq-\xi(t)^{2}\left|\frac{1+m_{1}}{1-K_{p}}\right|+\xi(t) m^{\top} M(t)+\xi(t) n z, \\
& \leq-c_{1} \xi(t)^{2}+c_{2} V(t)
\end{aligned}
$$

where $c_{1}$ and $c_{2}$ are obtained Cauchy Schwartz inequality and by completing the square. Finally, this yields :

$$
\begin{aligned}
\dot{W}(t) \leq\left(c_{2} r\left|K_{i}\right|-\omega_{1}\right) V(t)+\nu \mid & \left.K_{i} \xi\right|^{2} \\
& -r c_{1}\left|K_{i}\right| \xi(t)^{2}
\end{aligned}
$$

Picking $r\left|K_{i}\right|$ sufficiently small such that $c_{2} r\left|K_{i}\right|<\omega_{1}$ and $\left|K_{i}\right|$ sufficiently small yields the result.

\section{Simulation}

In this part, we implemented the PI controller on the nonlinear model (1)-(2), in a simulation. The numerical scheme used is a discretization in space of the equation (1) with 20 space nodes. The boundary conditions are then computed using ghost nodes for the spatial nodes out of the interval $[0 ; L]$. Values of the parameters are chosen following [6] and are given in Appendix A. The $\beta$ is supposed to model the possible frictions due to bore-hole grabbing or fluid/solid frictions. Considering the case of deep-water drilling system as in [9], we take $\beta=0.05 N$.s which corresponds to $\lambda \approx$ 0.3 . In the simulation, we consider the following scenario: Initial values are taken close to the equilibrium state such that a first regulation occurs. At $t=10 \mathrm{~s}$ the values of $T_{0}$ is increased by $50 \%$ during $1 \mathrm{sec}$ which leads to the apparition of the Stick-Slip phenomenon. In the case of a small $\left|\alpha_{p}\right|$ regulation is still effective, whereas, in the case of integral controller, regulation is not achieve due to the nonlinearity and so the mechanical oscillations go on. In Fig 1 we implement an integral controller with gains $k p=0$ and $k_{i}=0.2$, then $\alpha_{p}=-0.74$.In Fig 2 our gains are $k p=0.8$ and $k_{i}=0.2$, leading to $\alpha_{p} \approx-0.15$.

\section{CONCLUSION}

In this paper we have presented an analysis of the a PI controller to regulate the bottom velocity of a drill pipe. We have shown that exponential stability to the equilibrium could be achieved with this type of control laws. The result has been obtained employing a novel Lyapunov functional construction which is valid for all admissible mechanical parameters.

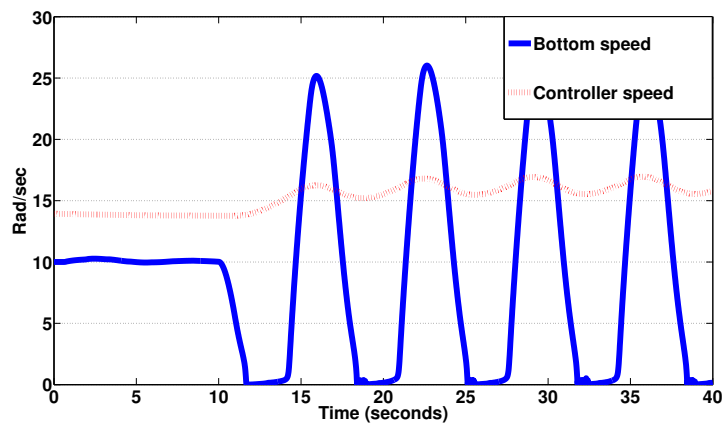

Fig. 1. Stabilization of the angular velocity

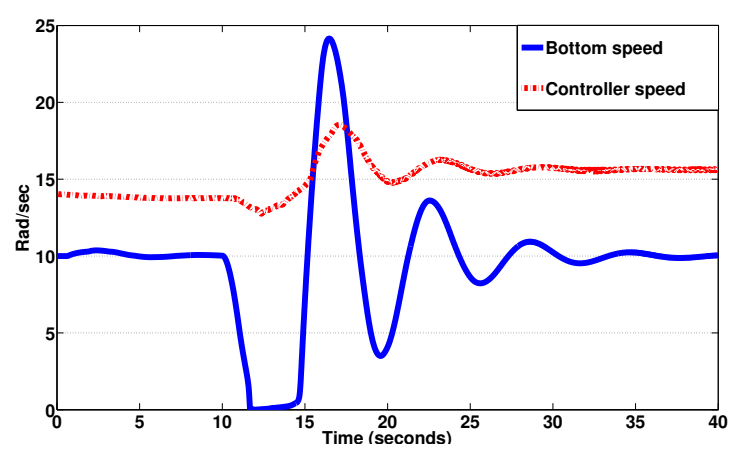

Fig. 2. Stabilization of the angular velocity

\section{REFERENCES}

[1] Jean Auriol, Jakob Ulf, Philippe Martin, and Florent Meglio. Delayrobust control design for two heterodirectional linear coupled hyperbolic pdes. arXiv preprint arXiv:1709.04274, 2017.

[2] Georges Bastin and Jean-Michel Coron. Stability and boundary stabilization of 1-d hyperbolic systems, volume 88. Springer, 2016.

[3] Halil Ibrahim Basturk. Observer-based boundary control design for the suppression of stick-slip oscillations in drilling systems with only surface measurements. Journal of Dynamic Systems, Measurement, and Control, 139(10):104501, 2017.

[4] Carlos Canudas-de Wit, Francisco R Rubio, and Miguel Angel Corchero. D-oskil: A new mechanism for controlling stick-slip oscillations in oil well drillstrings. IEEE Transactions on Control Systems Technology, 16(6):1177-1191, 2008.

[5] Valérie Dos Santos, Youssoufi Touré, Eduardo Mendes, and Estelle Courtial. Multivariable boundary control approach by internal model, applied to irrigation canals regulation. IFAC Proceedings Volumes, 38(1):63-68, 2005.

[6] JD Jansen and L Van den Steen. Active damping of self-excited torsional vibrations in oil well drillstrings. Journal of sound and vibration, 179(4):647-668, 1995.

[7] Martha Belem Saldivar Márquez, Islam Boussaada, Hugues Mounier, and Silviu-Iulian Niculescu. Analysis and Control of Oilwell Drilling Vibrations: A Time-Delay Systems Approach. Springer, 2015.

[8] Seppo Pohjolainen. Robust multivariable pi-controller for infinite dimensional systems. IEEE Transactions on Automatic Control, 27(1):17-30, 1982.

[9] Christophe Roman, Delphine Bresch-Pietri, Eduardo Cerpa, Christophe Prieur, and Olivier Sename. Backstepping observer based-control for an anti-damped boundary wave pde in presence of in-domain viscous damping. In Decision and Control (CDC), 2016 IEEE 55th Conference on, pages 549-554. IEEE, 2016.

[10] Conrad Sagert, Florent Di Meglio, Miroslav Krstic, and Pierre Rouchon. Backstepping and flatness approaches for stabilization of the stick-slip phenomenon for drilling. IFAC Proceedings Volumes, 46(2):779-784, 2013. 
[11] Ying Tang and Guilherme Mazanti. Stability analysis of coupled linear ode-hyperbolic pde systems with two time scales. Automatica, 85(Supplement C):386 - 396, 2017.

[12] Ngoc-Tu Trinh, Vincent Andrieu, and Cheng-Zhong Xu. Multivariable pi controller design for $2 \times 2$ systems governed by hyperbolic partial differential equations with lyapunov techniques. In Decision and Control (CDC), 2016 IEEE 55th Conference on, pages 5654-5659. IEEE, 2016

[13] Ngoc-Tu Trinh, Vincent Andrieu, and Cheng-Zhong Xu. Design of integral controllers for nonlinear systems governed by scalar hyperbolic partial differential equations. IEEE Transactions on Automatic Control, 2017.

[14] Cheng-Zhong Xu and Hamadi Jerbi. A robust pi-controller for infinitedimensional systems. International Journal of Control, 61(1):33-45, 1995.

[15] Cheng-Zhong Xu and Gauthier Sallet. Multivariable boundary pi control and regulation of a fluid flow system. Mathematical Control and Related Fields, 4(4):501-520, 2014.

\section{APPENDIX}

A. Mechanical parameters

\begin{tabular}{l|c|r} 
& Name & Values \\
\hline$G$ & Shear modulus & $79.6 \times 10^{9} \mathrm{~N} . \mathrm{m}^{-2}$ \\
$\rho$ & Mass density & $7850 \mathrm{~kg} \cdot \mathrm{m}^{3}$ \\
$J$ & Eq. mass moment of inertia & $1.19 \times 10^{-5} \mathrm{~m}^{4}$ \\
$\beta$ & Distributed damping & $0.05 \mathrm{~kg} . \mathrm{m} . \mathrm{s}^{-1}$ \\
$c_{a}$ & Torque transmitted & $2000 \mathrm{~N} . \mathrm{m} . \mathrm{s} . \mathrm{rad}^{-1}$ \\
$L$ & Length of the pipe & $2000 \mathrm{~m}$ \\
$I_{b}$ & BHA inertia & $311 \mathrm{~kg} . \mathrm{m}^{-2}$ \\
$c$ & Propagation speed & $3184.3 \mathrm{m.s}$ \\
$c_{b}$ & Dynamic friction & $0.03 \mathrm{~kg} . \mathrm{s}^{-1}$ \\
$T_{0}$ & Coulomb friction & $7500 \mathrm{~N}$ \\
\hline
\end{tabular}

